\title{
Neugeborenenscreening auf Mukoviszidose in Deutschland: Vergleich des neuen Screening-Protokolls mit einem Alternativprotokoll
}

\author{
Newborn Screening on Cystic Fibrosis in Germany: Comparison of \\ the new Screening Protocol with an Alternative Protocol
}

\section{Autoren}

Olaf Sommerburg',2, Mirjam Stahl1,2, Jutta Hammermann³, Jürgen G. Okun ${ }^{4}$, Andreas Kulozik ${ }^{1}$, Georg Hoffmann ${ }^{2,4 *}$, Marcus Mall1,2,5*

Institute

1 Sektion für Pädiatrische Pneumologie \& Allergologie und Mukoviszidose-Zentrum, Zentrum für Kinder- und Jugendmedizin, Universitätsklinikum Heidelberg

2 Zentrum für Translationale Lungenforschung Heidelberg (TLRC), Deutsches Zentrum für Lungenforschung (DZL), Universitätsklinikum Heidelberg

3 Klinik und Poliklinik für Kinder- und Jugendmedizin, Universitätsklinikum Dresden

4 Dietmar-Hopp-Stoffwechselzentrum, Zentrum für Kinder- und Jugendmedizin des Universitätsklinikums Heidelberg

5 Abteilung Translationale Pneumologie, Universitätsklinikum Heidelberg

Schlüsselwörter

Mukoviszidose, Neugeborenenscreening, genetisches Screening,

Pankreatitis assoziiertes Protein (PAP), Immunreaktives Trypsinogen (IRT)

Key words

cystic fibrosis, newborn screening, genetic screening, pankreatitis associated protein (PAP), immunoreactive trypsinogen (IRT)

Bibliografie

DOI http://dx.doi.org/10.1055/s-0042-124187

Klin Padiatr 2017; 229: 59-66

(c) Georg Thieme Verlag KG Stuttgart · New York

ISSN 0300-8630

Korrespondenzadresse

PD Dr. med. Olaf Sommerburg

Sektion für Pädiatrische Pneumologie \& Allergologie und

Mukoviszidose-Zentrum

Zentrum für Kinder- und Jugendmedizin

Universitätsklinikum Heidelberg

Im Neuenheimer Feld 430

69120 Heidelberg

Tel.: +49/6221/5638 790, Fax: +49/6221/5633 810

olaf.sommerburg@med.uni-heidelberg.de

Die Ergänzendes Material, Abb. E1, E2 und Tab.E1 des

Beitrags finden Sie Online unter http://dx.doi.org/

$10.1055 / \mathrm{s}-0042-124187$

\section{ZUSAMMENFASSUNG}

Hintergrund Zur Einführung des Neugeborenenscreening auf Mukoviszidose (CF) in Deutschland hat der Gemeinsame Bundesausschuss (G-BA) ein neues Screeningprotokoll mit der Bestimmung von Immunreaktiven Trypsinogen (IRT) als ersten und Pankreatitis assoziierten Protein (PAP) als zweiten Schritt eingeführt. In einem dritten Schritt folgt eine Analytik von 31 CFTR-Mutationen, um den positiven Vorhersagewert (PPV) gegenüber einem rein biochemischen IRT/PAP-Protokoll zu erhöhen.

Methoden Der Datenpool $(n=372$ 906) einer Studie zur Evaluation eines rein biochemischen IRT/PAP-Protokolls wurde für den Vergleich des vom G-BA beschlossenen Protokolls mit einem von den Autoren vorgeschlagenen Alternativprotokoll genutzt. Der Unterschied beider Protokolle liegt im weiteren Vorgehen, wenn das IRT >99,9. Perzentile liegt. Im G-BA-Protokoll wird dann sowohl der PAP- als auch der DNASchritt, im Alternativprotokoll nur der PAP-Schritt umgangen.

Ergebnisse Beide 3-stufigen Protokolle verlieren durch die DNA-Analytik im Vergleich zum 2-stufigen IRT/PAP-Protokoll nicht an Sensitivität. Unterschiede zeigten sich jedoch im PPV: Das G-BA-Protokoll zeigt mit 351 deutlich mehr falsch-positiv getestete Neugeborene (PPV 20,2\%) als das Alternativprotokoll mit 31 (PPV 69,6\%).

Schlussfolgerung Das G-BA-Protokoll hat im Vergleich zum Alternativprotokoll die schlechtere Performance. Durch die zu erwartende höhere Zahl an falsch-positiv detektierten Neugeborenen im G-BA-Protokoll werden mehr Vorstellungen zur Konfirmationsdiagnostik und für Schweißtests notwendig, mehr Familien verunsichert und höhere Kosten nach dem Screening verursacht.

\section{ABSTRACT}

Background For the new cystic fibrosis (CF) newborn screening program in Germany the Federal Joint Committee (G-BA) implemented a new screening protocol using immunoreactive trypsinogen (IRT) as first and pancreatitis associated protein (PAP) as second tier. Gene analysis with a panel of 31 CFTR-mutations is used as third tier to increase the positive predictive value (PPV) which is known to be low in pure biochemical IRT/PAP protocols. Methods For post hoc analysis the data pool $(n=372906)$ of a study evaluating a pure biochemical IRT/PAP protocol was used for assessment of the 3-step G-BA protocol in comparison with an alternative screening protocol recommended by the authors. The difference between the 2 protocols is the procedure when IRT $>99.9^{\text {th }}$ percentile. In the G BA protocol PAP and DNA analysis will be by-passed while in the alternative protocol only the PAP step will be circumvented.

Results Both 3-tier IRT/PAP + SN/DNA protocols did not lose sensitivity due to addition of genetic analysis when the results were compared to those of the 2-tier biochemical IRT/PAP protocol. However, the protocols provide different results regarding PPV. The G-BA protocol showed with

* geteilte Seniorautorenschaft 
351 a much higher number of false-positively detected newborns (PPV $20.2 \%$ ) when compared to 31 false-positively detected newborns in the alternative protocol (PPV 69.6\%).

Conclusions The G-BA protocol had a worse performance when compared with the alternative protocol recommended by the authors. The higher number of false-positively detected newborns using the G-BA protocol will lead to more consultations including sweat tests, will create more anxiety in parents, and will result in higher costs after screening.

\section{Einleitung}

Nach mehr als 8 Jahren Beratung wurde vom G-BA die Einführung des Neugeborenencreenings (NGS) auf Mukoviszidose (CF) beschlossen. Dieser Schritt war erwartet worden, weil in vielen entwickelten Ländern das CF-NGS seit langem zum Regelscreening gehört, sein Nutzen vielfach gezeigt wurde (u. a. [13]) und eine Studie zu Auswirkungen unterschiedlicher CF-NGS-Strategien in Deutschland bereits vor Jahren die Vorteile in diagnostischer und ökonomischer Hinsicht zeigen konnte [16]. Die lange Entscheidungsdauer war aber auch der schwierigen Diskussion geschuldet, welche die Einführung des Gendiagnostikgesetzes [1] vor seinem Inkrafttreten 2010 begleitet hatte. So war lange fraglich, ob in Deutschland ein Screening nach populationsspezifischen CFTRMutationen gesetzeskonform ist. Vor diesem Hintergrund wurde 2008 in den NGS-Zentren Heidelberg und Dresden mit Studien begonnen, in denen ein ursprünglich von Sarles et al. 2005 [18] publiziertes, rein biochemisches CF-NGS-Protokoll, basierend auf der Bestimmung des Immunreaktiven Trypsinogens (IRT) und des Pankreatitis assoziierten Proteins (PAP) evaluiert wurde. Beide Zentren verwendeten im Unterschied zum Originalprotokoll jedoch ein IRTabhängiges „Safety net“ (SN, IRT/PAP + SN) [23], weil nur so eine vergleichbare Sensitivität gegenüber modernen IRT/DNA-Protokollen erreicht werden konnte [21, 22]. In Heidelberg wurde das Originalprotokoll zudem derart modifiziert, dass statt 2 IRT-abhängiger PAP-Grenzwerte nur ein PAP-Grenzwert verwendet wurde [23]. Generelle Vorteile von IRT/PAP- gegenüber IRT/DNA-Protokollen sind die geringere Detektion von gesunden Heterozygoten und von Kindern mit unklarer CF-Diagnose (CFSPID), die nicht Ziel eines CF-NGS sind [9, 12, 21, 22, 26]. Nachteil rein biochemischer IRT/PAP- im Vergleich zu IRT/DNA-Protokollen ist jedoch ein schlechterer positiver Vorhersagewert (PPV) [9, 12, 21, 22, 26]. 2012 schlug deshalb eine niederländische Arbeitsgruppe vor, das IRT/PAP-Protokoll mit der Suche nach populationsspezifischen CFTR-Mutationen als dritten Schritt zu kombinieren, wodurch der niedrige PPV angehoben werden kann [26]. Diesen Vorteil erkannte auch der G-BA, der deshalb das Heidelberger IRT/PAP + SN-Protokoll [22, 23] mit der Suche nach 31 CFTR-Mutationen kombinierte. Neu am G-BA-Protokoll ist allerdings, dass bei einem IRT über der 99,9. Perzentile (SN) nicht nur keine PAP-, sondern auch keine DNA-Analytik durchgeführt wird, weil offenbar nur so ein Kompromiss mit dem aktuellen deutschen Gendiagnostikgesetz gefunden werden konnte [4]. Stattdessen erhalten alle Neugeborenen (NG), die durch das SN positiv getestet wurden, direkt die Konfirmationsdiagnostik einschließlich der Chlorid-Messung im Schweiß (

In der vorliegenden post-hoc Analyse wurde anhand der in Heidelberg generierten Daten das vom G-BA festgelegte CF-NGS-Protokoll evaluiert und mit einem von den Autoren vorgeschlagenen Alternativprotokoll verglichen. Letzteres ist nach derzeitiger Kom- mentierung wahrscheinlich nicht mit dem aktuellen Gendiagnostikgesetz vereinbar, stellt jedoch die logische Kombination aus dem Heidelberger IRT/PAP + SN-Protokoll und der Suche nach 31 CFTRMutationen als dritten Protokollschritt dar ( $\triangleright$ Abb. 1b).

\section{Patienten}

Die verwendeten Daten von 372906 gescreenten NG wurden von April 2008 bis April 2015 im NGS-Zentrum Heidelberg erhoben. Das Studienprotokoll der Originalstudie wurde durch die Ethikkommissionen Heidelberg sowie die der Ärztekammern Baden-Württembergs, des Saarlandes und Rheinland-Pfalz geprüft. Teilnehmende Eltern willigten nach Information schriftlich in die Studie ein. Da das für die Heidelberger Studie verwendete, rein biochemische IRT/PAP + SN- Protokoll bereits mehrfach beschrieben wurde [21-23], verweisen wir auf die Originalpublikationen bzw. die ausführliche Methodenbeschreibung im Online Supplement. Positiv getestete NG wurden in lokale CF-Zentren zur Chlorid-Messung im Schweiß [nach [5, 6]] und zur klinischen Evaluation überwiesen. Konnte eine CF nicht eindeutig diagnostiziert oder ausgeschlossen werden, erfolgte eine erweiterte Mutationsanalytik und/oder die Untersuchung der CFTR-vermittelten Chloridsekretion durch Kurzschlussstrommessung am Rektumschleimhautbiopsat $[7,8,10]$.

\section{Methode}

Das vom G-BA beschlossene 3-stufige CF-NGS-Protokoll wurde mit einem von den Autoren vorgeschlagenen 3-stufigen Alternativprotokoll verglichen ( $\triangleright$ Abb. 1a, b). Beide Protokolle folgen bis zum zweiten Schritt dem IRT/PAP + SN-Protokoll von Sommerburg et al. $[22,23]$. Als dritter Schritt folgt die Analytik der vom G-BA festgelegten 31 CFTR-Mutationen [4]. Einschließlich der vom Panel ausgeschlossen CFTR-Mutationen mit variabler Klinik (VCC) R117H und D1152H, umfassen diese 95,3\% der Allelfrequenz aller in Deutschland gefundenen CFTR-Mutationen [4]. Der Unterschied beider Protokolle liegt in der Wirkweise des SN. Während im G-BA-Protokoll alle SN-positiven NG bereits als CF-NGS-positiv gewertet werden, ohne dass der zweite und dritte Protokollschritt (PAP und DNA) zur Ausführung kommt, gilt im Alternativprotokoll der Autoren das SN nur für den PAP-Schritt ( $\triangleright$ Abb. 1a, b).

\section{Post-hoc Analyse}

Anhand der aus der Heidelberger Originalstudie vorhandenen IRTund PAP-Werte sowie den CFTR-Mutationen für die während und nach der Originalstudie diagnostizierten CF-Patienten (einschließlich der bekannten, falsch-negativ getesteten) wurde die Wirkweise der beiden 3-stufigen Protokolle für jedes der gescreenten NG nach- 


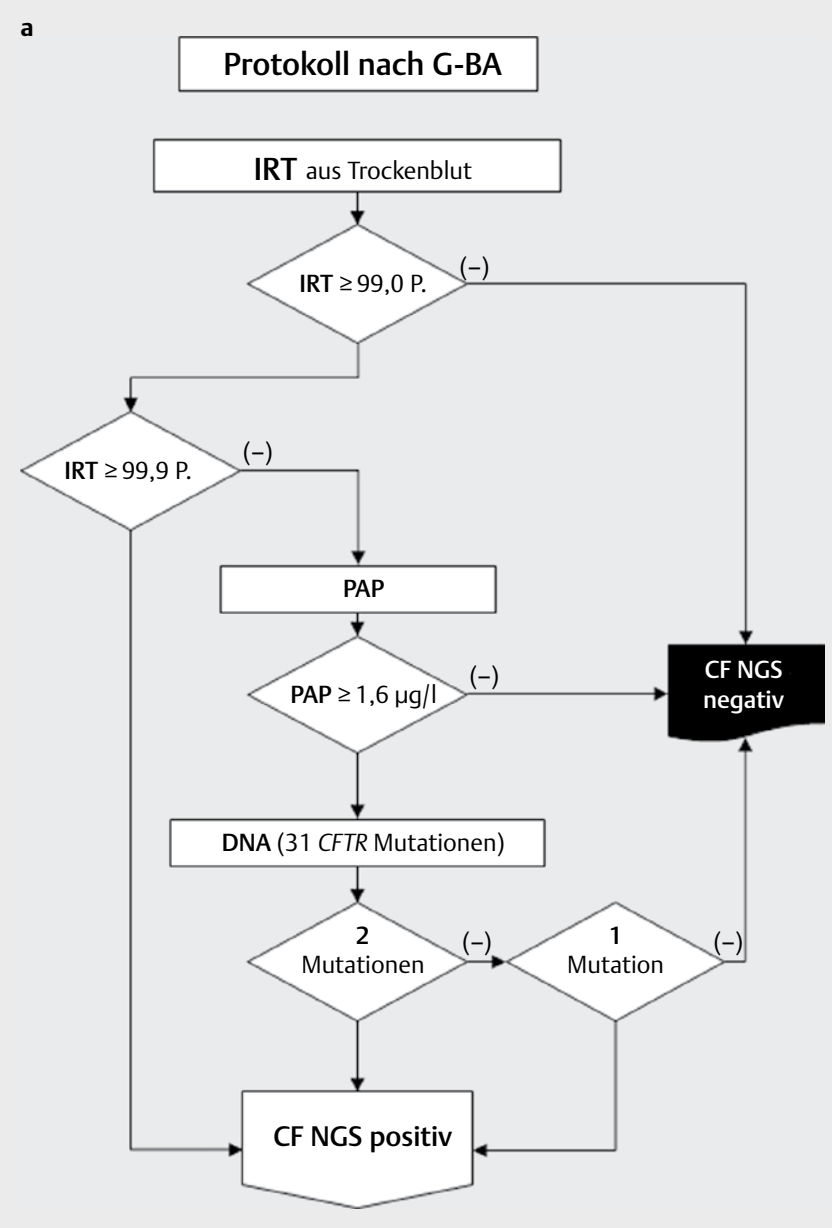

b
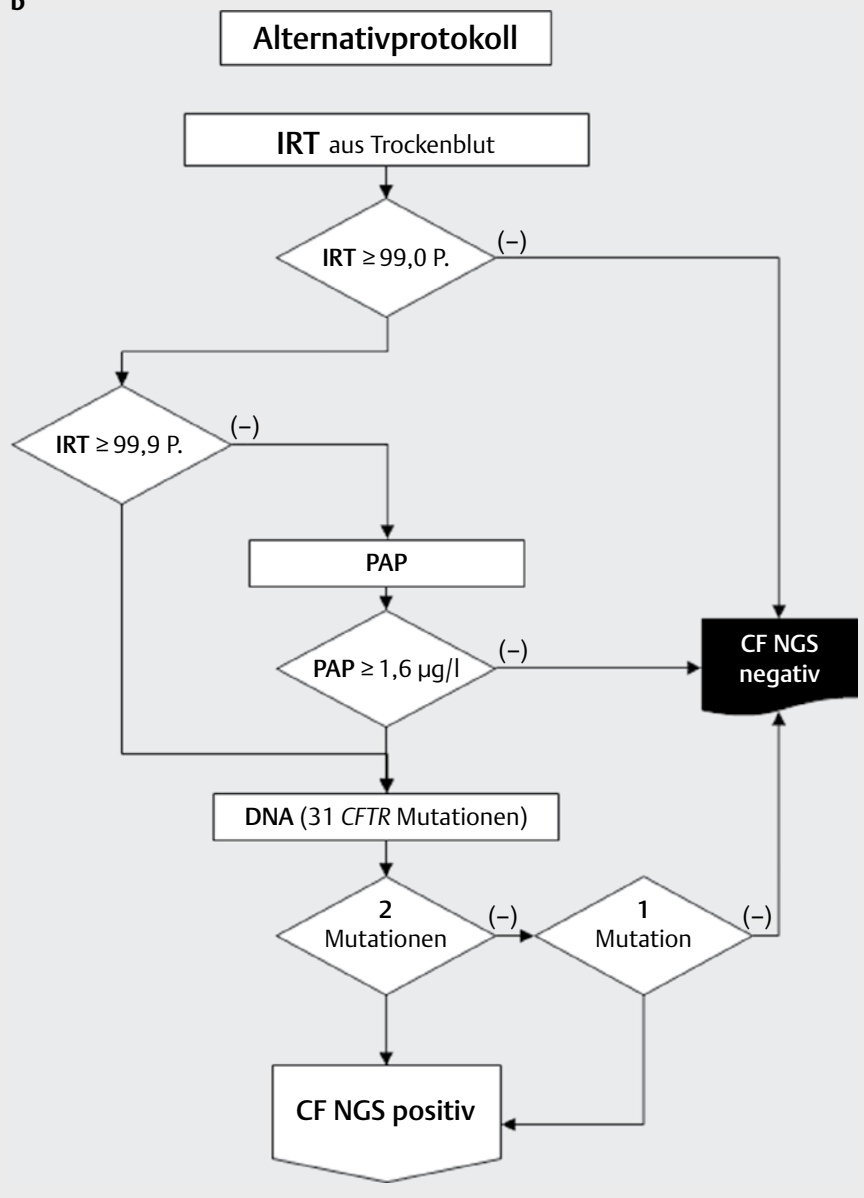

Abb. 1 a) Protokoll für das Neugeborenenscreening auf Mukoviszidose wie vom Gemeinsamen Bundesausschuss (GBA) beschlossen: Werden die Kriterien für das Safety Net (SN, IRT >99,9. Perzentile) erfüllt, werden keine PAP- und keine DNA-Analytik durchgeführt. b) Alternativprotokoll der Autoren: Bei Erfüllung der SNKriterien wird keine PAP-Analytik durchgeführt, jedoch erhalten die SN-positiven Neugeborenen eine DNA-Analytik.

vollzogen. Einzig die Anzahl der nach dem DNA-Schritt jeweils zu erwartenden NG, welche gesund aber heterozygot für eine pathologische CFTR-Mutation sind, konnte nicht direkt aus dem vorhandenen Daten geschlussfolgert werden, sondern musste mittels der HardyWeinberg-Gleichung $\left[(p+q)^{2}=p 2+2 p q+q 2\right]$ abgeschätzt werden. Dabei wurde zunächst der Anteil für die gesamte Studienpopulation berechnet. Für die IRT-positiv getesteten NG, wurde der dafür berechnete Anteil mit 2,5 multipliziert, da aus der Literatur bekannt ist, dass die Zahl der heterozygoten NG unter IRT-positiven Kindern 2bis 3-mal höher ist als der der Gesamtpopulation [15, 20]. Die so erhaltene Zahl heterozygoter NG wurde dann für die nach den einzelnen Protokollschritten jeweils positiv getesteten NG heruntergebrochen. Der Anteil heterozygoter NG nach dem PAP-Schritt wurde zudem um $75 \%$ reduziert, weil eine entsprechend geringe Detektionsrate heterozygoter NG in Studien beschrieben wurde, die IRT/ PAP- mit IRT/DNA-Protokollen verglichen hatten $[9,23,26]$. Die erhaltenen Resultate wurden zusammen mit den Ergebnissen der posthoc Analyse verwendet, um die Performance des G-BA- und des Alternativprotokolls der Autoren miteinander zu vergleichen. Zur besseren Einordnung der Resultate für die 3-stufigen Protokolle wurden diese zudem mit den Ergebnissen des rein biochemischen IRT/ PAP+SN-Protokolls aus der Heidelberger Studie [22, 23] verglichen.
Statistik Die Daten der CF-NGS Kohorte wurde in einer Microsoft Access 2010 Datenbank geführt. Die statistischen Berechnungen wurden mit Microsoft Excel 2010 und der aktuellen, freien, R“ Software „DTComPair“ durchgeführt [25]. Patienten mit Mekoniumileus, die ohnehin nach Geburt auffallen, Kinder mit CFSPID, die nicht Ziel eines CF-NGS sind, sowie ein frühgeborenes Kind, welches zwar ein initiales jedoch kein CF-Screening nach der korrigierten 32. SSW erhalten hatte, wurden von der statistischen Berechnung ausgeschlossen $[2,11]$.

\section{Ergebnisse}

Von April 2008 bis April 2015 wurden in Heidelberg 372906 Neugeborene mit dem IRT/PAP+SN-Protokoll auf CF gescreent. 2716 $(0,73 \%)$ NG waren IRT-positiv und erhielten die PAP-Analytik. Von diesen hatten $576(0,15 \%)$ ein PAP $>1,6 \mu \mathrm{g} / \mathrm{l}$ und $184(0,05 \%)$ der PAP-negativen NG waren SN-positiv, sodass insgesamt $760(0,20 \%)$ NG CF-NGS-positiv gewertet wurden ( $\bullet$ Abb. E1 im Online Supplement). 26 dieser Kinder waren bereits aufgrund anderer Erkrankungen als CF verstorben, sodass noch 734 NG einer Konfirmationsdiagnostik bedurften. Da die Eltern von 21 positiv getesteten NG $(2,8 \%)$ die Konfirmationsdiagnostik verweigerten, im Weiteren 
a

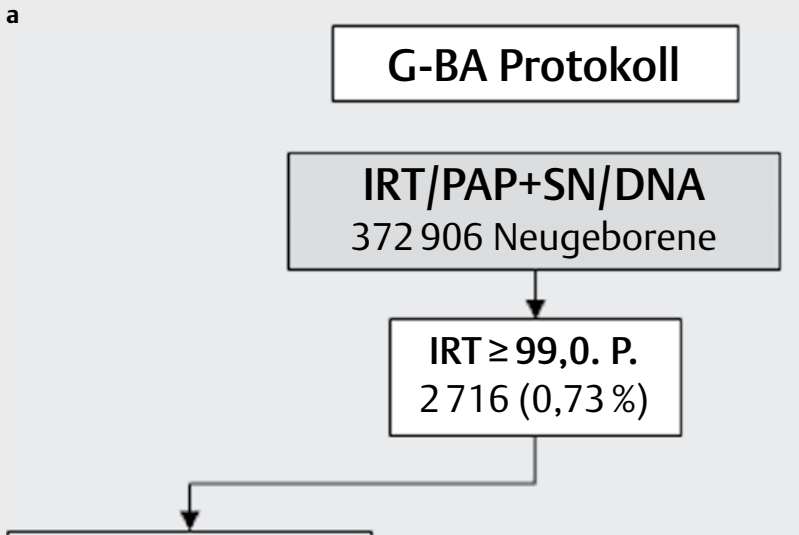

SN: IRT $\geq 99,9 . P . \quad(-)$ $322(0,09 \%)$

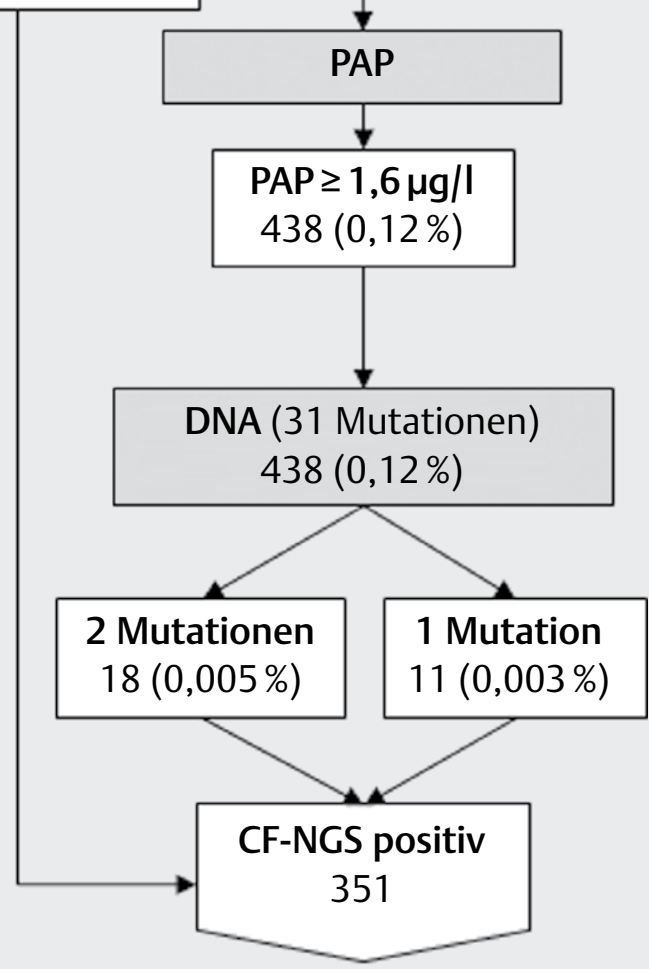

CF-Patienten

71 b

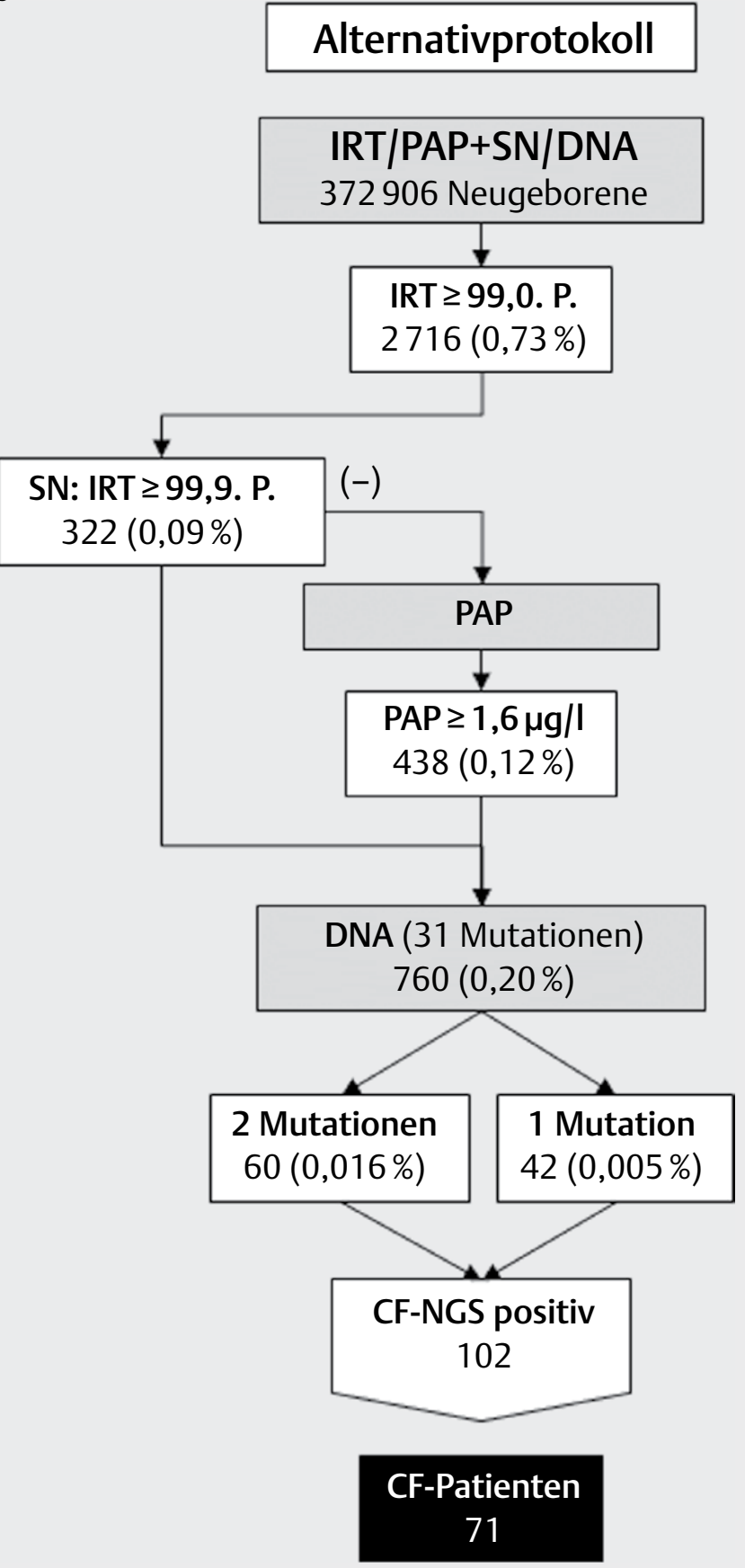

Abb. 2 Ergebnisse der beiden CF-NGS-Protokolle für das Neugeborenenscreening auf Mukoviszidose. a vom G-BA beschlossenes Protokoll, b von den Autoren vorgeschlagenes Alternativprotokoll.

die Eltern von 37 NG (4,9\%), die telefonisch nicht erreichbar waren, auf unsere per Einschreiben nachweislich zugestellte, postalische Kontaktaufnahme nicht antworteten, und zudem zu Eltern von 31 NG (4,1\%) trotz mehrfacher Versuche der Kontakt nach dem CFNGS nicht mehr hergestellt werden konnte (lost to follow-up), gelang die korrekte Durchführung der Konfirmationsdiagnostik nur bei 645 NG. Hierdurch wurden 71 CF-Patienten diagnostiziert.

- Tab. E1 im Online Supplement enthält die Screeningergebnisse (IRT, PAP, CFTR-Mutationen) sowie klinische Angaben (u. a.
Schweißtestbefund) aus der Heidelberger Originalstudie zu allen 71 CF-Patienten.

\section{Post-hoc-Analyse}

Die Resultate der post-hoc Analyse für beide 3-stufigen Protokolle sind in > Abb. 2 dargestellt. Die für jeden Protokollschritt kalkulierten Zahlen der zu erwartenden gesunden NG, die heterozygot für eine pathologische CFTR-Mutation sind, sind in der $>$ Abb. E2 im Online Supplement zu finden. 
- Tab. 1 Ergebnisse des vom G-BA beschlossenen CF-Screeningprotokolls für das Mukoviszidosescreening (A) im Vergleich zu den Ergebnissen eines von den Autoren vorgeschlagenen Alternativprotokolls (B).

\begin{tabular}{|c|c|c|}
\hline & \multicolumn{2}{|c|}{ Südwestdeutsche Population gescreent im Heidelberger Screeningzentrum von 2008 bis 2015} \\
\hline Neugeborene auf CF gescreent & \multicolumn{2}{|c|}{372906} \\
\hline Diagnostizierte CF-Patienten oder CFSPID gesamt & \multicolumn{2}{|c|}{79} \\
\hline Prävalenz & \multicolumn{2}{|c|}{ 1:4720 } \\
\hline Screeningprotokoll & Post hoc Analyse nach G-BA Protokoll & Post hoc Analyse nach Alternativprotokoll der Autoren \\
\hline Positive getestete Neugeborene & 351 & 102 \\
\hline Heterozygote & 8 & 31 \\
\hline CF detektiert ohne MI & 58 & 58 \\
\hline CF detektiert mit MI & 12 & 12 \\
\hline CFSPID & 1 & 1 \\
\hline Falsch-positiv & 280 & 31 \\
\hline CF-NGS-negativ & 372555 & 372804 \\
\hline Richtig-negativ & 372551 & 372800 \\
\hline Falsch-negativ, CF ohne MI & $4^{\#}$ & $4^{\#}$ \\
\hline Falsch-negativ, CF mit MI & 0 & 0 \\
\hline Negativ getested, CFSPID & 4 & 4 \\
\hline Sensitivität, \% (95\% Cl) & $96,0(88,8-98,6)$ & $96,0(88,8-98,6)$ \\
\hline Spezifität, \% (95\% Cl) & $99,9(99,9-99,9)$ & $100,0(100,0-100,0)$ \\
\hline PPV (95\% Cl) & $20,2(16,4-24,8)$ & $69,6(60,1-77,7)$ \\
\hline Verhältnis CF/CF-NGS positive NG & $1 / 4,9$ & $1 / 1,4$ \\
\hline \multicolumn{3}{|c|}{$\begin{array}{l}\text { \# Ein CF-Patient war ein Frühgeborenes, von dem nur eine Guthriekarte untersucht wurde, welche im Gestationsalter der korrigierten } 30 \text {. Schwanger- } \\
\text { schaftswoche abgenommen wurde. Dieser CF-Patient wurde nicht detektiert, weil keine zweite Guthriekarte nach der } 32 \text {. Schwangerschaftswoche } \\
\text { zum Screening auf CF zur Verfügung stand, wie das für Frühgeborene auch für alle anderen Screeningkrankheiten vorgeschrieben ist. Da es sich } \\
\text { hierbei um einen technischen bzw. logistischen Fehler handelte und nicht auf das verwendete Protokoll zurückzuführen ist, wurde dieses NG von den } \\
\text { statistischen Berechnungen ausgeschlossen }\end{array}$} \\
\hline
\end{tabular}

In beiden 3-stufigen Protokollen würden von den 2716 IRT-positiv getesteten NG 322 NG (0,09\%) die SN-Kriterien erfüllen und hätten deshalb im Unterschied zur Originalstudie keine PAP-Analytik erhalten. Während im G-BA-Protokoll ( $>$ Abb. 2a) diese NG bereits als CF-NGS-positiv gelten, würde man im Alternativprotokoll ( $\mathbf{A} \mathbf{A b}$. $\mathbf{2 b}$ ) bei diesen im dritten Protokollschritt noch die Suche nach den 31 CFTR-Mutationen durchführen. Bei alle anderen IRT-positiven NG würde sowohl im G-BA- als auch im Alternativprotokoll die PAP-Analytik durchgeführt werden, wonach jeweils 438 $(0,12 \%)$ einen PAP-Wert > 1,6 $\mu \mathrm{g} / \mathrm{I}$ gehabt hätten. Da im G-BA-Protokoll ( $\triangleright$ Abb. 2a) nur bei diesen NG die Mutationsanalytik erfolgt wäre, hätte man hier 18 NG mit 2 CFTR-Mutationen gefunden, die später auch als CF-Patienten diagnostiziert worden wären. Eine CFTR-Mutation wäre wahrscheinlich bei 11 NG gefunden worden, wobei sich später bei $3 \mathrm{NG}$ die CF in der Konfirmationsdiagnostik bestätigt hätte. Von den 322 NG (0,09\%) die die SN-Kriterien erfüllt hatten, wären nach der Konfirmationsdiagnostik bei 50 NG die CFDiagnose bestätigt worden. Somit hätten nur 30 \% der CF-Patienten im G-BA-Protokoll die CFTR-Mutationsanalytik erhalten. Insgesamt waren im G-BA-Protokoll 351 NG CF-NGS-positiv ( terschied dazu hätten im Alternativprotokoll ( $\triangleright \mathbf{A b b}$. 2b) sowohl die PAP- als auch die SN-positiven NG die Mutationsanalytik durchlaufen. Danach wären bei 60 NG 2 CFTR-Mutationen und bei 42 NG eine CFTR-Mutation nachgewiesen worden, sodass insgesamt 102 NG CF-NGS-positiv gewesen wären. Von diesen wären 71 NG, 60 NG mit Nachweis von 2 CFTR-Mutationen und 11 der 42 NG mit Nachweis einer CFTR-Mutation, nach der Konfirmationsdiagnostik als CF-Patienten diagnostiziert worden. Somit hatten alle 71 CFPatienten die Mutationsanalytik des CF-NGS erhalten. - Tab. 1 vergleicht die Ergebnisse des 3-stufigen G-BA- und des 3-stufigen Alternativprotokolls. Zur besseren Einordnung der Resultate verweisen wir zudem auf die Ergebnisse des 2-stufigen, rein biochemischen IRT/PAP + SN-Protokolls, welches ebenfalls 71 CF-Patienten detektiert hätte ( $\triangleright$ Abb. E1 im Online Supplement). Damit wäre eine Sensitivität von 96,0\% erzielt worden. Die gleiche Sensitivität zeigte sich auch nach der post-hoc Analyse für die beiden 3-stufigen Protokolle ( $\triangleright$ Tab. 1). Bezüglich der falsch-positiv getesteten NG gibt es jedoch Unterschiede: Im 2-stufigen, rein biochemischen Protokoll waren dies 689, im 3-stufigen G-BA-Protokoll 280 und im 3-stufigen Alternativprotokoll 31 NG. Dementsprechend war der PPV für das 3-stufige G-BA-Protokoll mit 20,2\% nur wenig besser als der PPV des 2-stufigen, rein biochemischen IRT/PAP+SN-Protokolls mit $9,3 \%$. Im Gegensatz dazu betrug der PPV des Alternativprotokolls 69,6\%.

\section{Diskussion}

Die Einführung des CF-NGS in Deutschland stellt einen erheblichen Gewinn für die medizinische Versorgung der CF-Patienten in Deutschland dar. Ohne CF-NGS wurden die CF-Patienten zu spät diagnostiziert, fast immer lagen bereits irreversible Veränderungen in den Atemwegen vor. Mit CF-NGS ist es jetzt möglich, CF-Patienten nach ihrer Geburt zu erkennen und mit effektiven Therapiemaßnahmen chronische Veränderungen der Lunge hinauszuzögern. Das jetzt eingeführte CF-NGS-Protokoll des G-BA ist als 
Kompromiss mit dem derzeit gültigen Gendiagnostikgesetz und den Rahmenbedingungen in Deutschland zu verstehen. Mit der Wahl eines PAP-basierten Screening-Algorithmus legten die G-BAExperten Wert auf dessen in der Einleitung genannten Vorteile. Mit der Inkorporation des Heidelberger IRT/PAP + SN Protokolls [2123] in das G-BA-Protokoll wurden auch die Vorteile der Modifikationen gegenüber dem Originalprotokoll von Sarles et al. [18, 19] anerkannt. Der G-BA sah ebenfalls den Nachteil des zu geringen PPV bei einem rein biochemischen IRT/PAP-Protokoll und entschied sich deshalb mit der Detektion von 31 populationsspezifischen CFTR-Mutationen für einen dritten Protokollschritt. Theoretisch ist durch die dritte Stufe ein Sensitivitätsverlust des Screeningprotokolls möglich, wenn die Auswahl der CFTR-Mutationen zu sehr eingeschränkt wird. Vergleicht man jedoch die in der post-hoc Analyse erzielte Sensitivität der 3-stufigen Protokolle mit der des 2-stufigen, rein biochemischen IRT/PAP + SN-Protokolls, so wird deutlich, dass dies für die hier untersuchte Population nicht der Fall war ( $\triangleright$ Tab. 1).

Mit der Funktion des IRT-abhängigen SN im jetzt eingeführten Screeningprotokoll betraten die G-BA-Experten jedoch Neuland. Während die Autoren das SN bisher nur als Ergänzung für den PAPTest interpretiert hatten, ging der G-BA mit seiner Version, sowohl den PAP- als auch den DNA-Schritt zu umgehen, deutlich weiter. Maßgeblich für diese G-BA-Entscheidung war wohl, das Recht auf Nichtwissen nach einer genetischen Untersuchung konform zum aktuellen Gendiagnostikgesetz zu wahren [4]. Normalerweise bedeutet ein positiver CF-NGS-Befund nach Mutationsanalytik immer, dass mindestens eine CFTR-Mutation vorliegt. Im vom G-BA festgelegten Screeningprotokoll kann ein NG durch das IRT-abhängige SN aber auch „rein biochemisch“ positiv auf CF gescreent werden. Da bei der Ergebnismitteilung nicht angegeben wird, welcher Parameter des CF-NGS positiv war, wäre dadurch das Recht auf Nichtwissen auch im Falle des Vorliegens einer CFTR-Mutation gewahrt. Interessant ist in diesem Zusammenhang der Vergleich mit dem CF-NGS der Schweiz [17], wo ein im Screening gesehener Heterozygotenstatus gesunder Kinder ebenfalls nicht mitgeteilt werden darf. In der Schweiz nutzt man für das CF-NGS sogar das IRT/DNAProtokoll, hier sorgt jedoch eine zentrale Trackingstelle dafür, dass Eltern bzw. das die Konfirmationsdiagnostik durchführende CF-Zentrum einen möglicherweise vorliegenden Heterozygotenstatus nur dann mitgeteilt bekommen, wenn die Diagnose CF bestätigt wurde [17]. In diesem Zusammenhang stellt sich für beide Länder in Zukunft allerdings die Frage, ob gut informierte Eltern diesen Wissensstand so akzeptieren werden. Wenn ihr Kind im CF-NGS positiv, in der Konfirmationsdiagnostik aber negativ war, werden viele Eltern für die weitere Familienplanung wissen wollen, ob sie selbst ggf. Anlageträger für eine Erkrankung sind, die so schwerwiegend ist, dass dafür ein NGS eingeführt wurde. In den „Tragenden Gründen“ zur Kinderrichtlinie [4] finden sich jedoch noch weitere Gründe für die Art der Anwendung des SN im G-BA-Screeningprotokoll. So soll das SN Ergänzung zur Mutationsanalytik für den Fall sein, dass ein NG mit CF keine der 31 gesuchten CFTR-Mutationen aufweist, wie das bspw. bei Kindern mit Migrationshintergrund der Fall sein könnte. Hierzu ist jedoch anzumerken, dass das Panel mit 31 CFTR-Mutationen bereits weit gefasst ist und da für ein positives CF-NGS die Detektion nur einer CFTR-Mutation ausreicht, kann für eine deutsche Population von einer Detektionsrate von 95,3\% aus- gegangen werden. Kalkuliert man nun, dass ein Kind mit Migrationshintergrund homozygot oder compound heterozygot für die verbleibenden Mutationen ist und zieht man die niedrigere Prävalenz in einem Land wie z. B. der Türkei (1: 10000 nach [3]) mit in Betracht, so kann angenommen werden, dass die Geburt eines solchen CF-Kindes in Deutschland nur etwa alle 7 Jahre auftritt, wobei hier auch noch nicht sicher ist, ob eine solche Konstellation seltener Mutationen auch wirklich krankheitsauslösend ist (ausführliche Berechnung im Online Supplement). Im Vergleich dazu muss bei einer Sensitivität des IRT/PAP + SN-Protokolls von 96,0\% jedoch davon ausgegangen werden, dass $4 \%$ der CF-Patienten möglicherweise mit schwerem Krankheitsverlauf bereits durch den biochemischen Protokollteil nicht gefunden werden.

Angesichts der deutlichen Unterschiede zwischen den beiden 3-stufigen Protokollen bei der Anzahl der falsch-positiv detektierten NG stellt sich die Frage, ob die oben genannten Begründungen die Art der Anwendung des IRT-abhängigen SN im G-BA-Protokoll wirklich rechtfertigen oder ob die Nutzung des SN wie im Alternativprotokoll der Autoren und eine Nichtmitteilung der Heterozygoten-Befunde wie in der Schweiz nicht sinnvoller gewesen wäre. Stellt man die Anzahl der detektierten CF-Patienten den positivgetesteten NG gegenüber, so mussten im 2-stufigen, rein biochemische Protokoll der Heidelberger Studie 10,7 NG positiv gescreent werden, um einen CF-Patienten zu detektieren (PPV 9,3\%). Das G-BA-Protokoll zeigt zwar ein besseres Verhältnis mit einem CF-Patienten auf 4,9 positiv gescreente NG (PPV 20,2\%), liegt damit aber immer noch unter den Qualitätsanforderungen der European Society of Cystic Fibrosis, welche einen PPV von mindestens $30 \%$ fordert [24]. Demgegenüber erreicht das Alternativprotokoll der Autoren ein Verhältnis von einem CF-Patienten zu nur 1,4 positiv gescreenten NG (PPV 69,6\%), was eine sehr geringe Anzahl falsch-positiv detektierter NG darstellt. Um den praktischen Unterschied zu verstehen, sollte man sich vor Augen führen, dass ein positives Ergebnis im NGS bedeutet, dass Eltern in Angst versetzt werden, dass deren Kind eine schwerwiegende, lebensbegrenzende Erkrankung haben könnte. Während bei den meisten metabolischen Stoffwechselerkrankungen, für die ein NGS angeboten wird, durch nochmalige Bestimmung des betreffenden Parameters relativ schnell diagnostische Sicherheit hergestellt werden kann, ist das bei der CF häufig nicht der Fall. So sind bei sehr jungen Säuglingen trotz ausreichender Expertise häufig 2 und mehr Schweißtests notwendig, bis die CF bestätigt oder ausgeschlossen werden kann. Nach Erfahrung der CF-Zentren, die im Rahmen der CF-NGS-Studien Chlorid-Messungen im Schweiß zur Konfirmationsdiagnostik durchgeführt haben, kann sich dieser Prozess in Einzelfällen auch über Wochen hinziehen. Dazu muss auch angemerkt werden, dass möglicherweise auch zu erwarten ist, dass die Expertise und die Voraussetzungen für die leitliniengerechte Durchführung der Chloridmessung im Schweiß nicht in allen Kinderkliniken gegeben ist [14]. Aus Sicht der CF-Behandler ist es deshalb wichtig, die Zahl der falsch-positiv detektierten NG von Beginn an so niedrig wie möglich zu halten. Wie die Ergebnisse zeigen, hätte man dieses Ziel mit dem Alternativprotokoll besser erreicht als mit dem jetzt eingeführten G-BA-Protokoll. Es muss aber anerkannt werden, dass mit dem Alternativprotokoll die Kosten für das reine CF-NGS aufgrund der höheren Anzahl an CFTR-Mutationsanalysen höher wären als mit dem G-BA-Protokoll. Betrachtet man allerdings den Gesamtauf- 
wand einschließlich der Konfirmationsdiagnostik, wird schnell klar, dass die vielen falsch-positiv detektierten NG im G-BA-Protokoll durch die notwendig werdenden klinischen Vorstellungen und Schweißtests wahrscheinlich höhere Kosten verursachen werden. Zudem kann man auch die Frage nach dem Sinn der CFTR-Mutationsanalytik im dritten Protokollschritt des G-BA-Protokoll stellen, wenn letztendlich nur $30 \%$ der detektierten CF-Patienten eine genetische Untersuchung erhalten hatten, während bereits 70\% über das IRT-abhängige SN diagnostiziert wurden.

Unsere Studie hat einige Limitierungen, die die Gesamtaussage möglicherweise beeinflussen. So hatten wir in der Originalstudie angegeben, dass etwa 53 \% der Geburtskliniken im Einzugsbereich des Screeningzentrums Heidelberg das CF-NGS im Rahmen unserer Studie nicht angeboten hatten. Da jedoch diese Geburtskliniken zufällig über den Einzugsbereich verteilt waren und keine Präferenz für einen bestimmten Kliniktyp bestand, kann davon ausgegangen werden, dass der Bias bei der Auswahl der NG vernachlässigbar ist. Im Weiteren hatten wir angegeben, dass insgesamt $11,7 \%$ der initial positiv getesteten NG nicht die vorgesehene Konfirmationsdiagnostik erhalten konnten. Dieser Punkt könnte unsere Ergebnisse beeinflusst haben, allerdings halten wir auch das für wenig wahrscheinlich, da hier kein systematischer Fehler bezüglich der sozialen Verteilung dieser NG zu erheben war. Erstaunlich ist allerdings, dass sich offenbar 2/3 dieser Eltern bewusst gegen die Konfirmationsdiagnostik entschieden hatten. Diese Situation unterstreicht die Notwendigkeit eines effektiven Trackingsystems in Zukunft unabhängig vom eingesetzten Screeningprotokoll [27].

Zusammenfassend ist festzuhalten, dass die Entscheidung des G-BA einen erheblichen Fortschritt für die Behandlung von CF-Patienten in Deutschland darstellt. Die Festlegung des CF-NGS-Protokolls durch den G-BA trägt den derzeitigen Rahmenbedingungen in Deutschland Rechnung. Durch Hinzunahme des Mutationsscreenings als dritten Protokollschritt konnte die Zahl der falsch-positiv detektierten Kinder gegenüber dem rein biochemischen PAP-Protokoll bereits gesenkt werden. Unsere Studie zeigt aber, dass mit dem Alternativprotokoll der Autoren die Zahl falsch-positiv detektierter NG noch einmal erheblich reduziert werden könnte. Mit der vom G-BA geforderten Evaluation des CF-NGS nach 3 Jahren besteht die Chance, ggf. notwendige Anpassungen des CF-NGSProtokolls umzusetzen.

\section{Stellungsnahme zur Autorenschaft}

O. Sommerburg: Contribution to study concept and design, acquisition of data, analysis and interpretation of data, drafting or revising the manuscript.

M. Stahl: Acquisition of data, analysis and interpretation of data, drafting or revising the manuscript.

J. Hammermann: Contribution to study concept and design, analysis and interpretation of data, drafting or revising the manuscript. J. Okun: Acquisition of data, analysis and interpretation of data, drafting or revising the manuscript.

A. Kulozik: Contribution to study concept and design, interpretation of data, drafting or revising the manuscript.
G. F. Hoffmann: Contribution to study concept and design, acquisition of data, analysis and interpretation of data, drafting or revising the manuscript.

M. A. Mall: Contribution to study concept and design, analysis and interpretation of data, drafting or revising the manuscript.

\section{Danksagung}

We gratefully thank all parents of newborns who participated in the study, all colleagues in participating obstetric and/or pediatric hospitals, Stephanie Hirtz (Heidelberg) and Margit Happich (Heidelberg) for technical assistance, and Julia Hentschel (Leipzig) and Gisela Haege (Heidelberg) for help in statistical analysis of the data. This study was sponsored by grants from the Dietmar-Hopp-Stiftung Walldorf GmbH (to O. S.) and BMBF (82DZL00401 and 82DZL004A1).

\section{Interessenkonflikt}

Die Autoren erklären hiermit, dass kein Interessenkonflikt besteht.

Literatur

[1] Anonymous. Gesetz über genetische Untersuchungen bei Menschen (Gendiagnostikgesetz - GenDG). In: BGBI. (ed) 2009: 2529

[2] Castellani C, Southern KW, Brownlee K et al. European best practice guidelines for cystic fibrosis neonatal screening J Cyst Fibros 2009; 8: 153-173

[3] Dayangac D, Erdem H, Yilmaz E et al. Mutations of the CFTR gene in Turkish patients with congenital bilateral absence of the vas deferens. Hum Reprod 2004; 19: 1094-1100

[4] Gemeinsamerbundesausschuss. Kinder-Richtlinie: Änderung des Beschlusses zur Neufassung - Screening auf Mukoviszidose (Zystische Fibrose) - Tragende Gründe zum Beschluss. 2015; https://www.g-ba. de/informationen/beschluesse/2316/; Letzter Zugriff: 07.10.2016

[5] Gibson LE, Cooke RE. A test for concentration of electrolytes in sweat in cystic fibrosis of the pancreas utilizing pilocarpine by iontophoresis. Pediatrics 1959; 23: 545-549

[6] Green A, Kirk J. Guidelines for the performance of the sweat test for the diagnosis of cystic fibrosis. Ann Clin Biochem 2007; 44: 25-34

[7] Hentschel J, Riesener G, Nelle H et al. Homozygous CFTR mutation M348K in a boy with respiratory symptoms and failure to thrive. Disease-causing mutation or benign alteration? Eur J Pediatr 2012; 171: 1039-1046

[8] Hirtz S, Gonska T, Seydewitz HH et al. CFTR Cl-channel function in native human colon correlates with the genotype and phenotype in cystic fibrosis. Gastroenterology 2004; 127: 1085-1095

[9] Krulisova V, Balascakova M, Skalicka V et al. Prospective and parallel assessments of cystic fibrosis newborn screening protocols in the Czech Republic: IRT/DNA/IRT versus IRT/PAP and IRT/PAP/DNA. Eur J Pediatr 2012; 171: 1223-1229

[10] Mall M, Kreda SM, Mengos A et al. The DeltaF508 mutation results in loss of CFTR function and mature protein in native human colon. Gastroenterology 2004; 126: 32-41

[11] Mayell SJ, Munck A, Craig JV et al. A European consensus for the evaluation and management of infants with an equivocal diagnosis following newborn screening for cystic fibrosis. J Cyst Fibros 2009; 8: 71-78 
[12] Mehta A, Sommerburg O, Southern KW. Newborn screening for cystic fibrosis: Opportunities and remaining challanges. In: Mall MA, Elborn JS. (eds) Cystic Fibrosis. European Respiratory Society. Sheffield: 2014: $65-76$

[13] Munck A, Dhondt JL, Sahler C et al. Implementation of the French nationwide cystic fibrosis newborn screening program. J Pediatr 2008; 153: $228-233$

[14] Naehrlich L. Durchführung und Interpretation des Schweißtests in deutschen Mukoviszidoseambulanzen. Klin Padiatr 2007; 219: 70-73

[15] Paracchini V, Seia M, Raimondi S et al. Cystic fibrosis newborn screening: Distribution of blood immunoreactive trypsinogen concentrations in hypertrypsinemic neonates. JIMD Rep 2012; 4: 17-23

[16] Rasch A, Perleth M. Modellierung der kurzfristigen diagnostischen und ökonomischen Auswirkungen eines Neugeborenen-screenings auf Mukoviszidose. Klin Padiatr 2011; 223: 96-103

[17] Rueegg CS, Kuehni CE, Gallati S et al. One-year evaluation of a neonatal screening program for cystic fibrosis in Switzerland. Dtsch Arztebl Int 2013; 110: 356-363

[18] Sarles J, Berthezene P, Le LC et al. Combining immunoreactive trypsinogen and pancreatitis-associated protein assays, a method of newborn screening for cystic fibrosis that avoids DNA analysis. J Pediatr 2005; 147: 302-305

[19] Sarles ], Giorgi R, Berthezene P et al. Neonatal screening for cystic fibrosis: comparing the performances of IRT/DNA and IRT/PAP. J Cyst Fibros 2014; 13: 384-390

[20] Scotet V, De Braekeleer M, Audrezet MP et al. Prevalence of CFTR mutations in hypertrypsinaemia detected through neonatal screening for cystic fibrosis. Clin Genet 2001; 59: 42-47
[21] Sommerburg O, Hammermann ], Lindner $\mathrm{M}$ et al. Five years of experience with biochemical cystic fibrosis newborn screening based on IRT/PAP in Germany. Pediatr Pulmonol 2015; 50: 655-664

[22] Sommerburg O, Krulisova V, Hammermann J et al. Comparison of different IRT-PAP protocols to screen newborns for cystic fibrosis in three central European populations. J Cyst Fibros 2014; 13: 15-23

[23] Sommerburg O, Lindner M, Muckenthaler M et al. Initial evaluation of a biochemical cystic fibrosis newborn screening by sequential analysis of immunoreactive trypsinogen and pancreatitis-associated protein (IRT/PAP) as a strategy that does not involve DNA testing in a Northern European population. J Inherit Metab Dis 2010; 33: S263-S271

[24] Southern KW, Munck A, Kashirskaya N. Newborn screening and access to specialist care from early life. 2013; https://www.ecfs.eu/files/ webfm/webfiles/File/Working\%20Groups/Neonatal\%20WG/ NSWG_newsletter_Aug2013.pdf; Letzer Zugriff: 07.10.2016

[25] Stock C, Hielscher T. DTComPair: Comparison of binary diagnostic tests in a paired study design. R package version 1.00. 2013; http:// CRAN.R-project.org/package = DTComPair; Letzter Zugriff: 07.10.2016

[26] Vernooij-Van Langen AM, Loeber JG, Elvers B et al. Novel strategies in newborn screening for cystic fibrosis: A prospective controlled study. Thorax 2012; 67: 289-295

[27] Winter T, Müller C, Schmidt S et al. 3 Jahre Mukoviszidose-Screening für Neugeborene in Mecklenburg-Vorpommern: Ein Erfahrungsbericht für die bundesweite Einführung. Monatsschr Kinderheilkd 2016; 164 : 591 